\title{
Heart Rate and Physical Activity Patterns in Persons With Profound Intellectual and Multiple Disabilities
}

\author{
Aly Waninge, ${ }^{1,2}$ Annette A.J. van der Putten, ${ }^{3}$ Roy E. Stewart, ${ }^{4}$ Bert Steenbergen, ${ }^{5}$ \\ Ruud van Wijck, ${ }^{6}$ and Cees P. van der Schans ${ }^{2,7}$ \\ ${ }^{1}$ Royal Dutch Visio De Brink, Vries, The Netherlands; ' Applied Sciences in Health Care and Nursing, Hanze University, \\ Groningen, The Netherlands; ${ }^{3}$ Department of Special Needs Education and Child Care, University of Groningen, Groningen, \\ The Netherlands; ${ }^{4}$ Public Health Groningen, Universal Medical Centre Groningen, University of Groningen, Groningen, The \\ Netherlands; ${ }^{5}$ Behavioural Science Institute Nijmegen, Radboud University Nijmegen, Nijmegen, The Netherlands; ${ }^{6}$ Center for \\ Human Movement Sciences, University Medical Center Groningen, University of Groningen, The Netherlands; and \\ ${ }^{7}$ Department of Rehabilitation Medicine, Center for Rehabilitation, University Medical Center Groningen, University of \\ Groningen, The Netherlands
}

\begin{abstract}
Waninge, A, van der Putten, AAJ, Stewart, RE, Steenbergen, $B$, Van Wijck, R, and van der Schans, CP. Heart rate and physical activity patterns in persons with profound intellectual and multiple disabilities. J Strength Cond Res 27(11): 31503158, 2013-Because physical fitness and health are related to physical activity, it is important to gain an insight into the physical activity levels of persons with profound intellectual and multiple disabilities (PIMD). The purpose of this study was to examine heart rate patterns to measure the activity levels of persons with PIMD and to analyze these heart rate patterns according to participant characteristics, observed level of activity, days, and time of day. The heart rate patterns of 24 participants with PIMD were measured continuously using a heart rate monitor for $8 \mathrm{~h} \cdot \mathrm{d}^{-1}$ for a period of 6 days. Physical activity levels were measured with questionnaires. Data were analyzed using multilevel analysis. The results indicate that the participants use only $32 \%$ of their heart rate reserve over 6 days. The intensity of heart rate reserve ranged from 1 to $62 \%$. On a given day, wide ranges in heart rates between participants and within persons were observed. Between days, only small ranges in the heart rate were found. The participants could be grouped into 4 classes according to their heart rate. In addition, factors such as time of day, physical activity, and age are significantly related to heart rate patterns. In conclusion, this study is an important first step in exploring activity patterns based on heart rate patterns in persons with PIMD. The participants used relatively small fractions
\end{abstract}

Address correspondence to Dr. Aly Waninge, alywaninge@visio.org. 27(11)/3150-3158

Journal of Strength and Conditioning Research

(C) 2013 National Strength and Conditioning Association

3150

Journal of Strength and Conditioning Research of their heart rate reserves. Time of day and age appear to have a considerable influence on heart rate patterns. The observed classes in heart rate patterns suggest that other probably more personal and psychosocial factors have significant influences on heart rate patterns, as well.

KEY WoRDS physical activity, persons with PIMD, sedentary, heart rate monitoring

\section{INTRODUCTION}

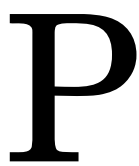

hysical activity maintains or improves mental health, physical health, physical fitness (3), and participation in daily life (World Health Organization) (35). Because people with profound intellectual, visual, and severe motor disabilities (profound intellectual and multiple disabilities [PIMD]) present the risk of low levels of physical activity $(9,17)$ and the associated negative effects on health, it is important to gain an insight into the physical activity levels of these persons.

Persons with PIMDs can be characterized as persons with such profound intellectual disabilities that no existing standardized tests are applicable for a valid estimation of their level of intellectual capacity. Therefore, their estimated intelligence quotient is $<20-25$ points (19), or their developmental level is $<24$ months (21). Because of the diffuse brain damage, they also possess profound neuromotor dysfunctions such as spastic tetraplegia. These individuals have little or no apparent understanding of verbal language, no apparent symbolic interactions with objects, and nearly no ability for selfsupport. In addition to the profound intellectual and severe or profound physical disabilities, sensory impairments are also frequently experienced (19). Comorbidity, such as epilepsy, gastroesophageal reflux, and chronic pulmonary infections, is more frequent in those with PIMD (26) compared with 
that in healthy subjects. In addition, it was recognized that persons with PIMD have a high chance of experiencing a variety of limitations in daily functioning, such as inactivity, insecure movement, and limited initiative (10).

The persons under study live in a residential care facility in the Netherlands, housing 200 persons with severe or PIMDs. Persons with PIMD living in such residential facilities are offered activities on a daily basis. From different studies, we know that these offered activities lack variation and are characterized as passive in nature, primarily aiming at offering sensory stimulation and under stimulating rather than motor activation (19). However, research into the physical activity levels in persons with PIMD is limited, and knowledge on this topic is scarce.

A possible reason for this gap in scientific knowledge could be that the physical activity tests that are regularly used in less severely disabled populations, such as the use of walking, are not applicable for persons with PIMD (33), because of the combination of profound intellectual, visual, and motor disabilities $(19,33)$. In general, persons with PIMD have notably limited mobility, use wheelchairs (19), and need others to enable them to be physically active. As a consequence, the presumed low levels of activity in persons with such profound disabilities are often not accurately presented by relatively insensitive measurement devices, such as activity monitors (33). In addition, there are no existing algorithms for predicting the activity energy expenditure of persons with PIMD.

Physical activity is defined as any bodily movement produced by skeletal muscles, which results in energy expenditure (5). The American College of Sports Medicine Position Stand (ACSM) (1) provides guidelines for healthy physical activity for the general population without disabilities. Physical activity studies often use a combination of assessment methods (13), including heart rate monitoring, which is an objective method (27), combined with direct observation, which is a criterion method (27). Therefore, in this study, the level of physical activity in persons with PIMD was determined by heart rate monitoring in combination with direct observation of physical activity.

The heart rate patterns of persons with PIMD, and the relationship between heart rate and the daily activity pattern for this specific group, have not been the subject of research to date. Additionally, the influence of likely covariates, such as gender and age, on the heart rate patterns of individuals with PIMD is unknown. Heart rate is related to age (2), gender, and activity (6). As persons with PIMD also suffer from comorbidities, such as motor disability, spasticity, and sensory disabilities, it seems useful to examine the influence of these covariates on heart rate as well. Furthermore, the possibility of a relationship between heart rate patterns and subgroups and time of day is suggested by the findings of Vlaskamp et al. (31). Their findings show that days in activity centers are highly structured, in a way that these persons are offered a fixed program in which activities are planned primarily between 10 and $11 \mathrm{AM}$ and between 2 and 3 PM (31).

In summary, our hypothesis is first that heart rate patterns in persons with PIMD will show that they are relatively inactive during regular days in the care facility. Second, our hypothesis is that age, gender, time of day, physical activity, and spasticity are related to heart rate patterns. Therefore, the purpose of this study is to explore heart rate and physical activity patterns in persons with PIMD. First, we monitor heart rate patterns in persons with PIMD, and second, we analyze heart rate patterns according to participant characteristics, observed level of activity, days, and time of day to establish a classification in heart-rate patterns.

\section{MethoDS}

\section{Experimental Approach to the Problem}

To test both our hypotheses, we determined the heart rate and physical activity patterns in persons with PIMD by monitoring the heart rate during a regular day in both the group home and the activity center. We made sure that there were no interventions other than "care as usual," to become a reliable impression of a regular day at the care facility, with regard to physical activity in particular.

In the activity center, one activity is offered to the participants in the morning, mostly between 10 and $11 \mathrm{AM}$, and 1 activity in the afternoon, between 2 and 3 PM. Physical activities that are offered are, for example, swimming, making movements with music, playing with toys with sound or a ball, lying on an air cushion or trampoline, while the care giver is jumping, active sitting on a bench without support instead of the own individually shaped wheelchair, gymnastics with a gymnastics instructor, and physical therapy. Other activities that are not assumed as physically activating are activities such as listening to music in their own wheelchair, lying on a water bed, and hand massage.

Physical activity studies often use a combination of assessment methods (13), including heart rate monitoring, which is an objective method (27), combined with direct observation, which is a criterion method (27). Because there is a relationship between activity intensity and the heart rate, heart rate monitoring may be used as a method to indicate activity levels (15). Moreover, heart rate monitoring appears to be sufficiently valid to be used in creating broad physical activity categories (e.g., highly active, somewhat active, sedentary) (24). Therefore, in this study, the level of physical activity in persons with PIMD was determined by heart rate monitoring in combination with direct observation.

To explore heart rate patterns, the heart rate was measured in each participant continuously for $8 \mathrm{~d}^{-1}$ for a period of 8 days. In parallel with heart-rate measurements, physical activities were registered with the use of a questionnaire, which was completed by a member of the support staff associated both with the living group and the activity center.

The quality of the heart rate data was checked, and artifacts were removed. In the end, 6 days per participant 
were left for further analysis of the heart beat data and the physical activity data. Five out of the 6 test days were weekdays; the remaining day was during a weekend. The measurements of all the participants were conducted randomly, across a 6-week period.

\section{Subjects}

The target population of our study comprises adults and adolescents with PIMD, as characterized by a profound intellectual disability indicated by an intelligence quotient $<20$ points or a developmental age $<24$ months (21) and are thus severely limited in terms of self-care, continence, communication, and mobility (23).

All the participants were recruited from a Dutch residential care facility, which houses 200 persons with severe or profound intellectual and visual disabilities. The inclusion criteria were the presence of a profound intellectual disability, visual impairment, and a severe or profound motor disability classified as Gross Motor Function Classification System level 4 or 5 (GMFCS, 20). The GMFCS is a 5-level system used to classify the locomotor skills of people with physical disabilities and is also applicable for persons with intellectual disabilities. The presence of a specific syndrome, like Down syndrome, was not an inclusion criterion.

For 48 persons, representatives were requested to provide written permission for participation in this study; written permission and consent were obtained from 30 individuals. A physician specialized in people with intellectual disabilities and a health care psychologist screened the participants for our exclusion criteria, which were severe psychological problems or somatic diseases, defined as chronic diseases and diseases that did not resolve over the short term. Four

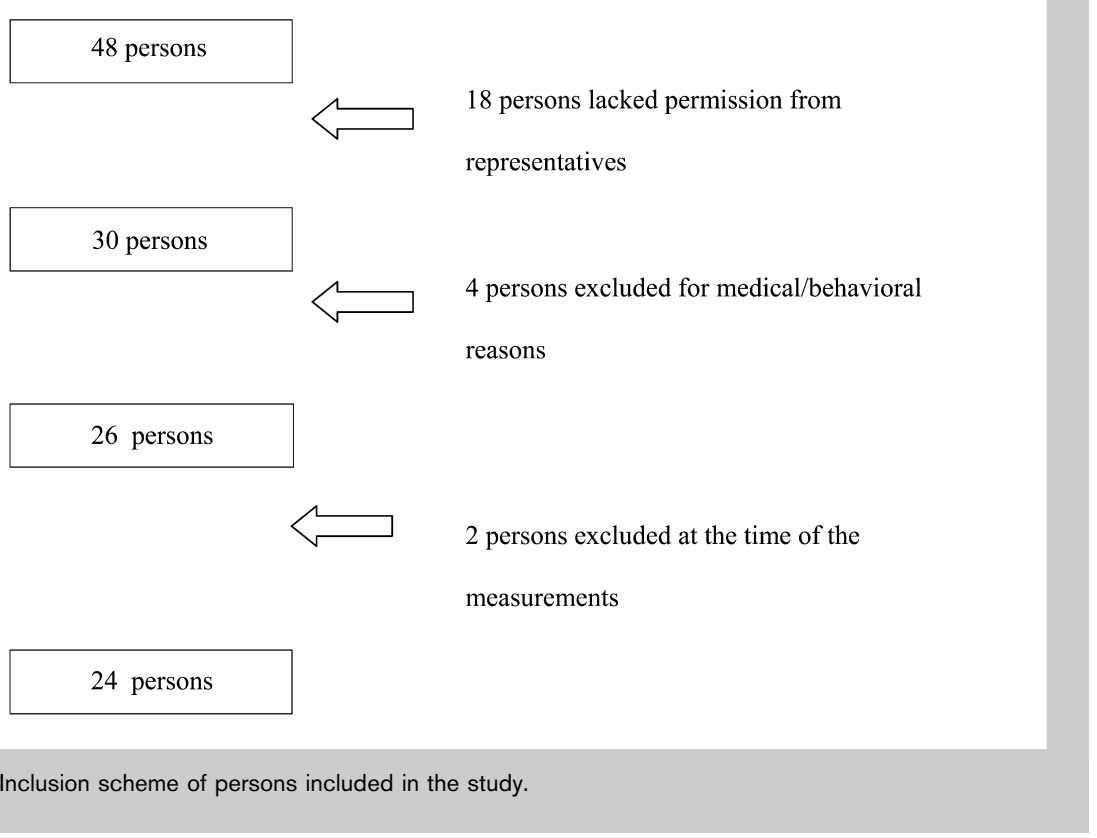

Figure 1. Inclusion scheme of persons included in the study. persons were excluded from the study because they showed one of these problems or diseases.

The exclusion criteria at the time of the measurements were general illness or fever; taking antibiotics; worsening of asthma or epilepsy (recent insult or epileptic fits); fresh wound(s) and bruise(s), or other factors causing pain during movement; stress because of the subject's behavior just before the measurement date; and current treatment with medication affecting heart rate, such as beta-blockers. Two persons were excluded because they presented one of these signals. Figure 1 presents the inclusion scheme.

All the participants were classified according to the GMFCS (20). The visual and auditory impairments of the participants were classified according to $\mathrm{WHO}$ guidelines (35): a distinction was made between being severely partially sighted and being partially sighted, and between severe hearing loss, slight hearing loss, and no hearing loss. Spasticity, if present, was classified as unilateral, bilateral, or unknown (8). Orthopedic defects were used as indicators of locomotor disabilities and were classified as present or not present. The use of medication was registered and classified as pain medication, medication for behavior or for muscle relaxation.

In all, 24 persons with PIMD participated in this study. Six women with a mean $(S D)$ age of $30(17)$ years participated; the mean $(S D)$ age of the men was $36(15)$ years. Table 1 shows the characteristics of the participants.

This study was performed in agreement with the guidelines of the Helsinki Declaration as revised in 1975. Permission to carry out the study was obtained from the institutional ethics committee. Informed consent was obtained from representatives of the participants because the participants were not able to provide their consent. The measurements were performed in accordance with the behavioral code section, titled "Resistance among people with an intellectual disability in the framework of the Act Governing Medical-Scientific Research Involving Humans" (behavioral code for physicians in the assessment of resistance among people with an intellectual disability) (7). Consistent distress or unhappiness was interpreted as a sign of lack of assent, and further participation in the study was reconsidered.

\section{Procedure}

Heart Rate Data. Heart rate patterns were measured with a Polar RS 800 heart rate monitor (Polar Electro Öy, 
TABLE 1. Characteristics of the participants.

\begin{tabular}{lcrrr}
\hline & & \multicolumn{2}{c}{ Gender } & \\
\cline { 2 - 3 } & & Male & Female & Total \\
\hline Visual impairments & Blind/severe & 13 & 3 & 16 \\
Auditory impairments & Partially & 5 & 3 & 8 \\
& Severe hearing loss & 2 & 0 & 2 \\
Orthopedic defects & Slight hearing loss & 3 & 0 & 3 \\
Spastic paresis & Normal hearing & 13 & 6 & 19 \\
& Yes & 16 & 6 & 22 \\
GMFCS level & No & 2 & 0 & 2 \\
Medication for behavior & Unilateral & 1 & 0 & 1 \\
Pain medication & Bilateral & 15 & 6 & 21 \\
Medication for muscle relaxation & No & 2 & 0 & 2 \\
& 4 & 5 & 1 & 6 \\
& 5 & 13 & 5 & 18 \\
& Yes & 14 & 4 & 18 \\
& No & 4 & 2 & 6 \\
& Yes & 4 & 4 & 8 \\
& No & 14 & 2 & 16 \\
& Yes & 6 & & 6 \\
& No & 12 & 0 & 18 \\
\hline
\end{tabular}

to the equation elaborated by Karvonen et al. (16), it is possible to compare these zones among participants.

Heart rate zones are calculated as follows. First, each participant's peak heart rate was estimated using the formula for participants with intellectual disabilities: 210-0.56 (age) - 15.5 (11). Because of the motor disabilities of the participants, no other noninvasive measurement of peak heart rate could be performed. Second, the participant's resting heart rate was determined by taking the median of 15 morning heart rate measurements taken before washing, toileting, and eating breakfast. Third, using

Kempele, Finland). Heart beat data were transferred offline to a computer. Once registered, the heart beat data were automatically stored using the Polar Pro Trainer 5 software supplied. The validity of Polar monitors in recording heart beat data has been confirmed against different types of electrocardiograms $(12,22,34)$.

Absolute peak heart rate, rest heart rate, and heart rate reserves differ for each person, which makes them difficult to compare directly. By calculating heart rate zones according participants' resting heart rate, the heart rate reserve was calculated by subtracting resting heart rate from estimated peak heart rate. Finally, the heart rate reserve was divided into 10 zones, with each zone consisting of $10 \%$ of the heart rate reserve. The continuous measurements of the heart rate are converted to heart rate zone per 15-minute interval, resulting in 32 calculated heart rate zones per day ( 8 hours, 4 times 15 minutes). For instance, a resting heart rate of $50 \mathrm{~b} \cdot \mathrm{min}^{-1}$, maximum heart rate of $180 \mathrm{~b} \cdot \mathrm{min}^{-1}$, heart rate reserve of 130 $\mathrm{b} \cdot \mathrm{min}^{-1}$, each heart rate zone consists of 13 heart rates. The first zone is from 50 to 62 ; the second is from 63 to 75; and so on. If the resting heart rate is $80 \mathrm{~b} \cdot \mathrm{min}^{-1}$ and the maximum heart rate is 150 $\mathrm{b} \cdot \mathrm{min}^{-1}$, then each heart rate zone consists of 7 heart rates per zone.

Physical Activity Data. In parallel with heart rate measurements, physical activities were registered using direct observation per 15 minutes over 8 $\mathrm{h} \cdot \mathrm{d}^{-1}$ for a period of 8 days. These physical activities were registered 


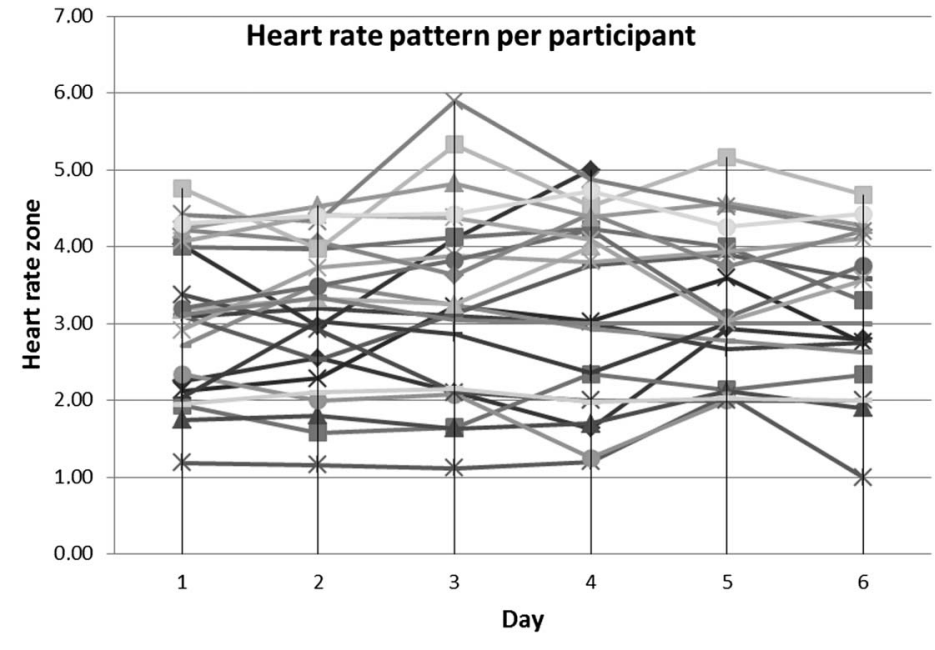

Figure 2. The mean heart rate zones of each participant $(n=24)$ over 6 days with a day-to-day outline of the mean prevalence of each heart-rate zone among the participants. Next, the means and ranges of the heart rate zones were calculated for the overall group. Furthermore, mean heart rate zones per participant of all 6 days are shown in a figure. Our hypothesis was that heart rate patterns in persons with PIMD will show that they are relatively inactive during the normal course of business in the care facility.

Heart Rate Patterns According to Days, Observed Level of Activity, Time of Day, Participant Characteristics, and Heart Rate Classes. Heart rate patterns according to using a questionnaire and noted in score lists by a member of the support staff associated both with the living group and the activity center. The reliability of the questionnaire was tested in an earlier study and seemed to be adequate. The agreement between the data obtained was acceptable with a Cohen's kappa value of 0.70 (28).

Physical activity was coded as targeted physical activity: "Yes" or "No." Moving with the wheelchair inside or outside, transferring, active sitting without support, gymnastics with a gymnastics instructor, physical therapy, playing with a ball, and "dancing" to music were all examples of targeted physical activity, "Yes." Listening to music, watching television, or lying down on a bed were all examples of targeted physical activity, "No."

\section{Statistical Analyses}

The data were analyzed using SPSS 16.0 and multilevel analysis using the Mlwin computer program (18). For all statistical tests, the alpha level for significance was $p \leq 0.05$.

Heart Rate Patterns. An overview of the heart rate zones was used to explore the heart-rate patterns in this study along

TABLE 3. Variance between participants, days, and time of day.

\begin{tabular}{lc}
\hline Random & \\
\hline Participant & 0.911 \\
Days & 0.161 \\
Time of day & 0.429 \\
Total variance & 1.501 \\
2Loglikelihood & 10302 \\
\hline
\end{tabular}

days: To gain an insight into the heart rate patterns of persons with PIMD, 3 decomposing variance components were used with a linear mixed model: (a) between persons, (b) within persons between days, and (c) within days. The mean heart rate and variance proportion component (VPC) were calculated between persons, within persons, and between days. As an indicator of variance in the heart rate zone, the VPC is calculated by dividing the variance by the total variance. Subsequently, the generalizability coefficient for the relative differences is calculated using the variance proportion (4). A generalizability coefficient of $\geq 0.80$ indicates sufficient reliability.

Heart rate patterns according to the level of physical activity, time of day, and participant characteristics: To determine how the heart rate relates to the level of physical activity, we estimated equations of physical activity as a variable dependent on heart rate. Furthermore, we examined the influence of the "time of day" on this relationship.

The influence of covariates, such as gender, age, time of day, daily activities, motor disabilities, spasticity, and sensory disabilities, were evaluated in the mixed model. Our hypothesis was that age, time of day, daily activities, and spasticity would have an influence on heart rate patterns.

Heart rate patterns and classes: To identify distinct groups of heart rate patterns and to examine these classes in the heart rate, we used a latent class analysis $(29,30)$. Because the dependent variable was a count variable, a Poisson distribution was used for this analysis.

\section{RESUlts}

\section{Heart Rate Patterns in Persons with PIMD}

Table 2 shows a day-to-day outline of the mean prevalence of heart rate zones in the participants, indicating that the participants did not reach heart rates $>55 \%$ of their heart rate reserves for a consecutive 30 minutes. 
TABLE 4. Linear mixed models of heart rate.*

\begin{tabular}{|c|c|c|c|c|c|c|c|c|}
\hline \multirow{2}{*}{$\begin{array}{l}\text { Fixed } \\
\text { (person level) }\end{array}$} & \multicolumn{2}{|c|}{ Model 0} & \multicolumn{2}{|c|}{ Model 1} & \multicolumn{2}{|c|}{ Model 2} & \multicolumn{2}{|c|}{ Model 3} \\
\hline & $\begin{array}{c}B \\
\text { value }\end{array}$ & $\begin{array}{l}\text { Standard } \\
\text { error }\end{array}$ & $\begin{array}{c}B \\
\text { value }\end{array}$ & $\begin{array}{c}\text { Standard } \\
\text { error }\end{array}$ & $B$ value & $\begin{array}{c}\text { Standard } \\
\text { error }\end{array}$ & $B$ value & $\begin{array}{c}\text { Standard } \\
\text { error }\end{array}$ \\
\hline Intercept & 3.196 & 0.198 & 3.185 & 0.198 & 4.091 & 0.303 & 4.217 & 0.655 \\
\hline $\begin{array}{l}\text { Time of day } \\
\text { Activity }\end{array}$ & & & & & $-0.007 \dagger$ & 0.001 & $-0.007 \dagger$ & 0.001 \\
\hline $\begin{array}{l}\text { Targeted activity vs. no } \\
\text { targeted (ref) }\end{array}$ & & & $0.041 \dagger$ & 0.021 & 0.021 & 0.021 & 0.021 & 0.021 \\
\hline Age & & & & & $-0.035 \dagger$ & 0.011 & $-0.023 \dagger$ & 0.012 \\
\hline Gender & & & & & & & & \\
\hline Male vs. female (ref) & & & & & & & -0.041 & 0.409 \\
\hline Orthopedic defects & & & & & & & & \\
\hline $\begin{array}{l}\text { No orthopedic defects vs. orth } \\
\text { defects (ref) }\end{array}$ & & & & & & & -0.257 & 0.577 \\
\hline Spastic pareses & & & & & & & & \\
\hline Bilateral vs. unilateral paresis (ref) & & & & & & & 0.499 & 0.867 \\
\hline Visual impairment & & & & & & & & \\
\hline $\begin{array}{l}\text { Severe partially vs. partially impaired } \\
\text { vision (ref) }\end{array}$ & & & & & & & 0.185 & 0.39 \\
\hline Auditory impairment & & & & & & & & \\
\hline $\begin{array}{l}\text { Severe hearing loss vs. slight } \\
\text { hearing loss }\end{array}$ & & & & & & & -0.606 & 0.593 \\
\hline $\begin{array}{l}\text { Slight hearing loss vs. normal } \\
\text { hearing }\end{array}$ & & & & & & & -0.735 & 0.462 \\
\hline
\end{tabular}

${ }^{*} B$ value $=$ the regression coefficient; Ref $=$ reference variable. $\dagger$ Means significant relation.

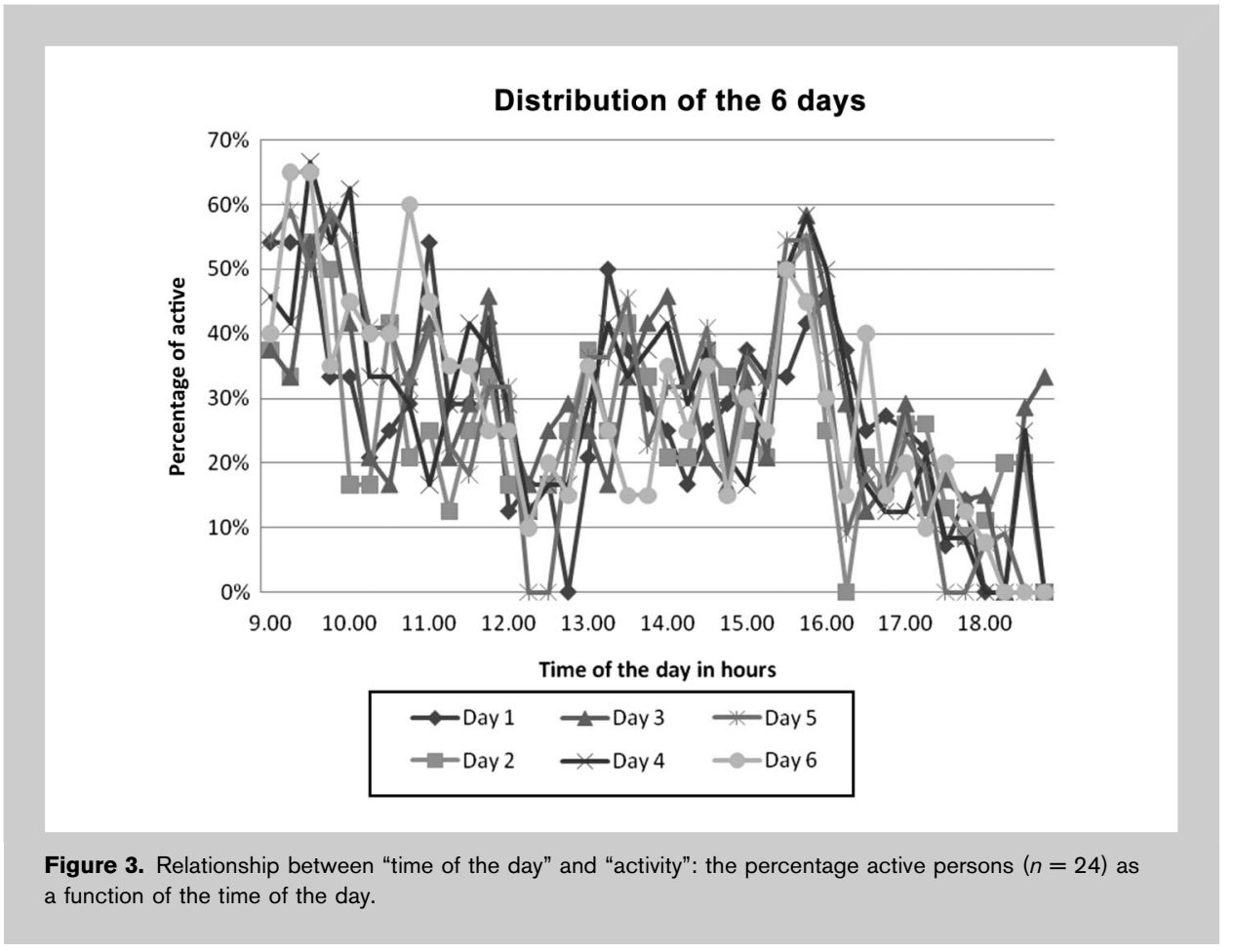

The mean heart rate zone over the 6 test days is shown under the heading "intercept" in model 0 in Table 4 . The value achieved was 3.196 , indicating $32 \%$ of the heart rate reserve. The results obtained range from 1 to $62 \%$ of the heart rate reserve.

Figure 2 visualizes the heart rate patterns of each participant over 6 days. It is shown that there is variance between the participants and variance between the days.

\section{Heart Rate Patterns According to Days, Observed Level of Activity, Time of Day, Participant Characteristics, and Heart Rate Classes} Heart Rate Patterns According to Days. Table 3 shows the variance between participants 
(0.911), between days (0.161), and within 1 day (0.429). Because the total variance is the sum of these variances (1.501), the VPC was $60.7 \%$ between participants, $10.7 \%$ between days, and 28.6\% among different times of day. These VPCs indicate that the variation between the test days is relatively low. The generalizability coefficient for the relative differences, as calculated with the VPCs, is 0.85 , indicating that heart rate monitoring is a reliable measurement.

Heart Rate Patterns According to Participant Characteristics, Level of Physical Activity, and Time of Day. Table 4 (model 3) shows the influence of covariates. Age has a significant influence. Higher ages yielded lower heart rate zones. Time of day also has a significant influence: lower heart rate zones were observed later on in the day. Gender, spastic pareses, intellectual level, and visual and hearing impairments had no significant influence.

The relationship between time of day and heart rate is significant (regression coefficient -0.007 with standard error (SE) $0.001 ; p<0.01$; see models 2 and 3 in Table 4$)$. The relationship between physical activity and heart rate in model 1 is presented in Table 4 with a regression coefficient of 0.041 with an $S E$ of 0.021 , which also represents a significant relationship $(p=0.02)$. However, if "time of the day" is brought into the equation, there is no longer any significant influence of "physical activity" on the heart rate (regression coefficient 0.022 with $S E 0.021 ; p=0.147$ ); only "time of day" remains as an influence (see model 2 in Table 4). Therefore, we examined the relationship between "time of day" and "daily activity" (Figure 3 ).

Figure 3 shows the percentage of active persons as a function of the time of day and illustrates that most participants are active during the first 8 quarters of the day (between 9.00 and $11.00 \mathrm{AM}$ ). During the day, the percentage of active persons decreases. Most participants seem to rest between the 13th and 16th quarters (between 12.15 and $13.00 \mathrm{PM}$ ). Most participants become active again from the 18th until the 28th quarter, but activity decreased again afterward.

Heart Rate Patterns and Classes. Because of the variability in age, time of day, and the observations measured on 6 different days, we found a 4-class solution that provides a clear clinical interpretation $(29,30)$. The 4 classes were high heart rate zone (class 1), middle stable heart rate zone (class 2 ), low heart rate zone (class 3 ), and variation in the middle heart rate zone (class 4 ).

\section{Discussion}

This study examined the heart-rate and physical activity patterns in participants with PIMD over an extended period during the day. Second, this study analyzed heart-rate patterns in relation to participant characteristics, level of activity, days, and time of day to establish the classification in terms of heart rate patterns.
Our first hypothesis was that persons with PIMD are relatively inactive during regular days in the care facility, as indicated by their heart rate patterns. This hypothesis is confirmed: the participants did not exhibit heart rates accounting for $>50 \%$ of their heart rate reserves for a consecutive 30 minutes. Furthermore, the results show that the mean heart rate zone of the participants over 6 days was 3.2, indicating only $32 \%$ of their heart rate reserves. The intensity ranged from 1 to $62 \%$ of their heart rate reserve. In addition, wide ranges in the heart rate between participants and also during the days in 1 participant were demonstrated. However, we found small ranges in the heart rate between days.

Our second hypothesis was that age, gender, time of day, physical activity, and spasticity are related to the heart rate patterns. This hypothesis can only be partly confirmed. Time of day, physical activity, and age have a significant influence on the heart rate. However, the relationship between time of day and physical activity is notably strong and when corrected for "time of day," "physical activity" ceases to have a significant influence on the heart rate. The participants could be classified into 4 groups according to their heart rate.

This study is an important first step in exploring the heart rate patterns of persons with PIMD. Because of the associated motor disabilities, examination of the peak heart rate with a maximal heart rate test is not possible. Therefore, we had to use the formula proposed by Fernhall et al. (11), which is an estimation and may therefore be less reliable. However, the results of this first exploration may be useful for practice. First, heart-rate monitoring provides an insight into the daily activity patterns of persons with PIMD. Second, heart-rate monitoring can be used to evaluate the interventions geared at promoting physical activity and participation in daily activities.

The mean heart rate zone of all the participants when examined for 6 days was 3.2. This indicates that the participants use relatively little, only $20-30 \%$, of their heart rate reserves. Although the range of percentages of heart rate reserves was found to be between 1 and $62 \%$, levels $>50 \%$ for at least a consecutive 30 minutes were not reached. A first explanation for not achieving these physical activity levels might be that the physiology of the subjects is different from that of less severely disabled populations. A second explanation might be that it is difficult to spur this study's participants to physical activity as a consequence of their limited skills in moving actively. The subjects are hindered by visual impairment and a lack of comprehension. Furthermore, as a consequence, persons with PIMD need others to enable them to be physically active. The individual combinations of these limitations may explain the 4 classes of heart rate patterns that we distinguished in this study. Future research should be directed toward the examination of these classes and the possible influence of interventions on these classes.

Because of the individual limitations of the persons and the fact that these persons make a group of case studies, we 
used multilevel analysis for the statistical analysis. Multilevel analysis makes it possible not only to compare between participants, but simultaneously to compare within a person between days and between different times of a day.

Caregivers in the living situation, and caregivers at the activity center, filled out the physical activity questionnaire that was used in our analysis. This information analyzed by Van der Putten en Vlaskamp (25) was similar to that provided by the heart rate patterns: the mean number of motor activities offered was 0.8 per day (range: $0-3.3 ; S D: 1.1$ ), and the mean duration of motor activity was $26 \mathrm{~min} \cdot \mathrm{d}^{-1}$ (range: $0-163$; $S D$ : 35) (25). This study yielded a negative correlation between the heart rate and age. Van der Putten and Vlaskamp (25) found a negative correlation between daily activity and age. Because heart rate height and physical activity are considered to be related (3), our results are in line with the results of Van der Putten and Vlaskamp (25).

Furthermore, the majority of observed motor activity took place during "daily care situations," whereas only $18 \%$ of these situations involved targeted motor activity. Sixty-one percent of the activities were passive in nature (32). Therefore, motor activity is a minor part of the support for persons with PIMD. Moreover, analyzing the heart rate patterns in this study shows that these individuals did not meet the ACSM recommendations (1) for health-related physical fitness. Haskell et al. (14) stated that "the greatest health benefits from an increase in activity appear to occur when very sedentary persons begin a regular program of moderate intensity, endurance-type activity." Based on our data, a lot of persons with PIMD are sedentary, and therefore, developing and performing interventions geared to promote physical activity in persons with PIMD is of the utmost importance.

The relationship between time of day and heart rate may be an illustration of the highly structured days at the activity centers. This finding is in line with the findings of Vlaskamp et al. (31): in 6 care facilities in the Netherlands, it seemed to be common practice to have one activity in the morning, mostly between 10 and $11 \mathrm{AM}$, and one activity in the afternoon, between 2 and 3 PM. This degree of structure already made us to expect a minimal difference in heart rate patterns between days. However, is such a structure desirable? Would an alternating-day rhythm result in more activity among people with PIMD? Notably, the advantage of the minimal difference in test results between days is that one day of testing suffices.

We found no significant relationship between heart rate and gender, intellectual level or comorbidity as motor disabilities, spasticity, and sensory disabilities when examining eight-hour heart rate patterns. To test for any possible significant relationship, future research could set up subcategories, controlling for groups with a specific comorbidity with groups without the specific comorbidity.

In conclusion, the participants did not achieve heart rates accounting for $>50 \%$ of their heart rate reserves for a consecutive 30 minutes and used relatively little of their heart rate reserves. Time of day and age have a considerable influence on heart rate patterns. However, the observed classes in heart rate patterns suggest that other, more personal factors have a significant influence on heart rate patterns, as well.

\section{Practical Applications}

The results of this first exploration may be useful for practice. First, heart rate monitoring reveals an insight into the daily activity patterns of persons with PIMD and may be used to identify sedentary persons. Second, heart rate monitoring can be used to evaluate interventions geared at facilitating physical activity and participation in daily activities. Third, developing and performing interventions geared to promote physical activity in persons with PIMD is of the utmost importance.

Developing these interventions, the following issues should be taken into account: (a) The interventions should focus on persons with PIMD of all ages, because the age of persons with PIMD is strongly related to the level of physical activity. (b) Daily structure in the activity centers is an important issue: we suppose that a more alternatingday rhythm would possibly result in more physical activity among people with PIMD. (c) The intervention should lead to a daily increase in the heart rate per person based on a baseline measurement of heart rate during 3 days. As Haskell et al. (14) described, "the greatest health benefits from an increase in activity appear to occur when very sedentary persons begin a regular program of moderate intensity, endurance-type activity." However, it will require a lot of creativity to develop such a program for persons with PIMD, because of their limited capacities in moving actively.

\section{ACKNOWLEDGMents}

This research was financed by Hanze University Groningen and Royal Dutch Visio De Brink. The authors kindly acknowledge and thank the participants and their representatives for their participation in this study and thank both personal caregivers at the living group and the support staff of the activity center of Royal Dutch Visio De Brink for filling out the questionnaires. Furthermore, the authors gratefully acknowledge and thank Floor J. Hettinga, $\mathrm{PhD}$, for advice on physiological topics. The authors report no conflict of interest or professional relationship with companies or manufacturers who will benefit from the results of this study. In addition, the results of this study do not constitute endorsement of the product by the authors or the National Strength and Conditioning Association.

\section{REFERENCES}

1. American College of Sports Medicine Position Stand. The recommended quantity and quality of exercise for developing and maintaining cardiorespiratory and muscular fitness, and flexibility in healthy adults. Med Sci Sports Exerc 30: 975-991, 1998.

2. Baynard, T, Pitetti, KH, Guerra, M, Unnithan, VB, and Fernhall, B. Age-related changes in aerobic capacity in individuals with mental retardation: A 20-yr review. Med Sci Sports Exerc 40: 1984-1992, 2008. 
3. Bouchard, C, Shepard, RJ, and Stephens, T. Physical Activity, Fitness and Health. Champaign, IL: Human Kinetics Publishers, 1994.

4. Brennan, RL. Generalizability Theory. New York, NY: SpringerVerlag, 2001.

5. Capsersen, CJ, Powell, KE, and Christenson, GM. Physical activity, exercise, and physical fitness: Definitions and distinctions for healthrelated research. Public Health Rep 100: 127-131, 1985.

6. Climstein, M, Pitetti, KH, Barrett, PJ, and Campbell, KD. The accuracy of predicting treadmill $\mathrm{VO}_{2}$ max for adults with mental retardation, with and without Down's syndrome, using ACSM gender- and activity-specific regression equations. J Intellect Dis Res 37: 521-531, 1993.

7. Dutch Society for Physicians in the Care for People With an Intellectual Disability (NVAZ). Resistance among people with an intellectual disability in the framework of the act governing medicalscientific research involving humans, behavioural code for doctors in the assessment of resistance among people with an intellectual disability, 1999.

8. Dutch Society for Physicians in Rehabilitation. Protocol for diagnosis and treatment of children with cerebral palsy and spasticity, 2006.

9. Emerson, E. Underweight, obesity and exercise among adults with intellectual disabilities in supported accommodation in Northern England. J Intellect Dis Res 49: 134-143, 2005.

10. Evenhuis, HM, Sjoukes, L, Koot, HM, and Kooijman, AC. Does visual impairment lead to additional disability in adults with intellectual disabilities? J Intellect Dis Res 53: 19-28, 2009.

11. Fernhall, B, McCubbin, JA, Pitetti, KH, Rintala, P, Rimmer, JH, Millar, AL, and De Silva, A. Prediction of maximal heart rate in individuals with mental retardation. Med Sci Sports Exerc 33: 16551660, 2001.

12. Gamelin, FX, Berthoin, S, and Bosquet, L. Validity of the polar S810 heart rate monitor to measure R-R intervals at rest. Med Sci Sports Exerc 38: 887-893, 2006.

13. Guinhouya, BC, Apété, GK, and Hubert, H. Evaluation of habitual physical activity of children during clinical and epidemiological trials. Sante Publique 21: 465-478, 2009.

14. Haskell, WL, Lee, IM, Pate, RR, Powell, KE, Blair, SN, Franklin, PA, Macera, CA, Heath, GW, Thompson, PD, and Bauman, A. Physical activity and public health updated recommendation for adults from the American College of Sports Medicine and the American heart Association. Circulation 116: 1081-1093, 2007.

15. Hunter, J. Energy costs of wheelchair propulsion by elderly and disabled people. Int J Rehab Res 10(4 Suppl 5):50-54, 1987.

16. Karvonen, MJ, Kentala, E, and Mustala, O. The effects of training on heart rate: A longitudinal study. Annu Med Exp Fenn 35: 307-315, 1957.

17. Lahtinen, U, Rintala, P, and Malin, A. Physical performance of individuals with intellectual disability: A 30 year follow up. Adapt Phys Act Q 24: 125-143, 2007.

18. Multilevel Models Project: A User's Guide to MLwiN. [Computer Program], Version 2.10. London: University of Bristol, 2004.

19. Nakken, $\mathrm{H}$ and Vlaskamp, C. A need for taxonomy for profound intellectual and multiple disabilities. J Policy Pract Int Dis 4: 83-87, 2007.
20. Palisano, R, Hanna, SE, Rosenbaum, PL, Rusell, DJ, Walter, SD, Wood, EP, Raina, PS, and Galuppi, BE. Validation of a model of gross motor function for children with cerebral palsy. Phys Ther 80: 974-985, 2000.

21. Pawlyn, J and Carnaby, S. Profound Intellect Multiple Disabilities: Nursing Complex Needs (Vol. 6). West Sussex, United Kingdom: Blackwell Publishing Ltd., 2009.

22. Porto, LG and Junqueira, LF Jr. Comparison of time-domain shortterm heart interval variability analysis using a wrist-worn heart rate monitor and the conventional electrocardiogram. Pacing Clin Electrophysiol 32: 43-51, 2009.

23. Schalock, R, Brown, I, Brown, R, Cummins, RA, Felce, D, Matikka, L, Keith, KD, and Parmenter, T. Conceptualization, measurement, and application of quality of life for persons with intellectual disabilities: Report of an international panel of experts. Ment Retard 40: 457-470, 2002.

24. Sirard, JR and Pate, RR. Physical activity assessment in children and adolescents. Sports Med 31: 439-454, 2001.

25. Van der Putten, AAJ and Vlaskamp, C. Motor activation in people with PIMD. J Appl Res Intellect Dis 23: 496, 2010.

26. Van Schrojenstein Lantman-de Valk, HMJ, Metsemakers, JFM, Haveman, MJ, and Crebolder, HFJM. Health problems in people with intellectual disability in general practice: a comparative study. Fam Pract 17: 405-407, 2000.

27. Vanhees, L, Lefevre, J, Philippaerts, R, Martens, M, Huygens, W, Troosters, T, and Beunen, G. How to assess physical activity? How to assess physical fitness? J Cardiovasc Prev Rehab 12: 102-114, 2005.

28. Verheijen, JAT and Brussee, FHP. Motor activation: a study into the motor activation of persons with profound intellectual and multiple disabilities. Masterthesis, University of Groningen, The Netherlands, 2009.

29. Vermunt, JK and Magidsen, J. Latent class models for classification. Comput Stat Data Anal 41: 531-537, 2003.

30. Vermunt, JK, Tran, B, and Magidsen, J. Latent class models in longitudinal research. In: Handbook of Longitudinal Research: Design, Measurement, and Analysis. S. Menard, ed. Burlington, MA: Elsevier, 2007. pp. 373-385.

31. Vlaskamp, C, Hiemstra, SJ, Wiersma, LA, and Zijlstra, BJH. Extent, duration, and content of day services' activities for persons with profound intellectual and multiple disabilities. J Policy Pract Intellect Dis 4: 152-159, 2007.

32. Vlaskamp, C and Van der Putten, AAJ. Physical activities in people with PIMD: When and how are they offered. J Appl Res Intellect Dis 23: 496, 2010.

33. Warms, C. Physical activity measurement in persons with chronic and disabling conditions, methods, strategies and issues. Fam Community Health 29: 788-799, 2006.

34. Weippert, M, Kumar, M, Kreuzfeld, S, Arndt, D, Rieger, A, and Stoll, R. Comparison of three mobile devices for measuring R-R intervals and heart rate variability: polar S810i, Suunto t6 and an ambulatory ECG system. Eur J Appl Physiol 109: 779-786, 2010.

35. World Health Organisation. International Classification of Functioning, Disability and Health. Geneva, Switzerland: WHO, 2001. 